MATHEMATICS OF COMPUTATION

Volume 71 , Number 237 , Pages $27-47$

S $0025-5718(01) 01313-8$

Article electronically published on May 14, 2001

\title{
PROJECTION METHOD III: SPATIAL DISCRETIZATION ON THE STAGGERED GRID
}

\author{
WEINAN E AND JIAN-GUO LIU
}

\begin{abstract}
In E \& Liu (SIAM J Numer. Anal., 1995), we studied convergence and the structure of the error for several projection methods when the spatial variable was kept continuous (we call this the semi-discrete case). In this paper, we address similar questions for the fully discrete case when the spatial variables are discretized using a staggered grid. We prove that the numerical solution in velocity has full accuracy up to the boundary, despite the fact that there are numerical boundary layers present in the semi-discrete solutions.
\end{abstract}

\section{INTRODUCTION}

In this paper, we continue our study of convergence and error structure for the projection method. In previous work [3], we studied semi-discrete situations when the temporal variable is discretized, but the spatial variable is kept continuous. We proved that as far as velocity is concerned, the method of Chorin and Temam 2, 12 is uniformly first order accurate up to the boundary, and the second order method of 6, 9] is uniformly second order accurate up to the boundary. However, due to the presence of numerical boundary layers, pseudo-pressure has only half order of accuracy near the boundary. We characterized explicitly the structure of the numerical boundary layer, and proved that full accuracy for pressure can be recovered if we subtract the numerical boundary layer profiles from the numerical solution. We also showed, using normal mode analysis, that the boundary layer modes turn into oscillatory modes for the pressure-increment formulation of [13, 1]. For a summary of these results, we refer to [4]. For related convergence results for the semi-discrete case, we refer to [10, 11].

The presence of singular modes in the semi-discrete solutions raises serious doubts on the convergence of the fully discrete method. Indeed, it has been known for some time that accuracy and even convergence can be lost if care is not exercised at the projection step. This is documented carefully in [5]. It has also been known for some time that full accuracy is kept if the spatial discretization is done on a staggered grid. This paper is devoted to a proof of this empirical fact.

Our work still leaves open the very important issue of characterizing the minimum condition the spatial discretization has to satisfy in order to guarantee accuracy. The working assumption seems to be that as long as the projection step is truly a projection, i.e. the numerical projection operator $P_{h}$ satisfies: (1) $P_{h}$ is

Received by the editor May 19, 1997 and, in revised form, March 1, 2000.

2000 Mathematics Subject Classification. Primary 65M06, 76M20.

Key words and phrases. Viscous incompressible flows, projection method, numerical boundary layer, finite difference, convergence. 
self-adjoint; (2) $P_{h}^{2}=P_{h}$ (we will refer to this as the projection condition), accuracy in velocity will be kept. It remains to be seen whether this is true in general. We refer to [16] for some progress in this direction. On the other hand, there are many situations in which enforcing the projection condition is difficult. Therefore we are motivated to seek numerical methods which do not require the projection condition. The gauge method [5] has so far proved to be a very attractive alternative in this regard.

This paper is organized as follows. In the next section, we review the projection method, with emphasis on spatial discretization on the staggered grid (also known as the MAC grid [7]). In Section 3, we summarize our main results. In Sections 4 and 5 we present the proof for the first and second order methods respectively.

\section{REVIEW OF THE PROJECTION METHODS}

In primitive variables, the Navier-Stokes equation (NSE) takes the form

$$
\left\{\begin{array}{l}
\partial_{t} \boldsymbol{u}+(\boldsymbol{u} \cdot \nabla) \boldsymbol{u}+\nabla p=\Delta \boldsymbol{u}, \\
\nabla \cdot \boldsymbol{u}=0 .
\end{array}\right.
$$

Here $\boldsymbol{u}=(u, v)$ is the velocity, $p$ is the pressure. For simplicity, we will only consider the case when the no-slip boundary condition (BC) is supplemented to (2.1):

$$
\boldsymbol{u}=0 \quad \text { on } \partial \Omega,
$$

where $\Omega$ is an open domain in $\boldsymbol{R}^{2}$ with smooth or piecewise smooth boundary.

2.1. Time discretization. The first order projection method of [2, 12] proceeds in two steps.

Step 1: Computing the intermediate velocity field $\boldsymbol{u}^{*}$ :

$$
\left\{\begin{array}{l}
\frac{\boldsymbol{u}^{*}-\boldsymbol{u}^{n}}{\triangle t}+\left(\boldsymbol{u}^{n} \cdot \nabla\right) \boldsymbol{u}^{n}=\Delta \boldsymbol{u}^{*} \\
\boldsymbol{u}^{*}=0, \quad \text { on } \partial \Omega
\end{array}\right.
$$

Step 2: Projecting to the space of divergence-free vector fields to obtain $\boldsymbol{u}^{n+1}$ :

$$
\left\{\begin{array}{l}
\boldsymbol{u}^{*}=\boldsymbol{u}^{n+1}+\Delta t \nabla p^{n+1} \\
\nabla \cdot \boldsymbol{u}^{n+1}=0
\end{array}\right.
$$

The projection step enforces the boundary condition:

$$
\boldsymbol{u}^{n+1} \cdot \boldsymbol{n}=0, \quad \text { or } \quad \frac{\partial p^{n+1}}{\partial \boldsymbol{n}}=0, \quad \text { on } \partial \Omega .
$$

Second order schemes. We will concentrate on the formulation in [6, 9], and refer to 4 for a summary of other second order methods. We have

$$
\left\{\begin{array}{l}
\frac{\boldsymbol{u}^{*}-\boldsymbol{u}^{n}}{\Delta t}+\left(\boldsymbol{u}^{n+1 / 2} \cdot \nabla\right) \boldsymbol{u}^{n+1 / 2}=\Delta \frac{\boldsymbol{u}^{*}+\boldsymbol{u}^{n}}{2} \\
\boldsymbol{u}^{*}+\boldsymbol{u}^{n}=\Delta t \nabla p^{n-1 / 2}, \quad \text { on } \partial \Omega \\
\boldsymbol{u}^{*}=\boldsymbol{u}^{n+1}+\Delta t \nabla p^{n+1 / 2}, \\
\nabla \cdot \boldsymbol{u}^{n+1}=0, \\
\boldsymbol{u}^{n+1} \cdot \boldsymbol{n}=0, \quad \text { on } \partial \Omega .
\end{array}\right.
$$


In this formulation, the homogeneous Neumann BC for pressure,

$$
\frac{\partial p^{n+1 / 2}}{\partial \boldsymbol{n}}=0, \quad \text { on } \partial \Omega,
$$

is retained. An inhomogeneous BC for $\boldsymbol{u}^{*}$ is introduced so that the slip velocity of $\boldsymbol{u}^{n+1}$ at the boundary is of order $(\triangle t)^{2}$.

Remark. The nonlinear convection term $\left(\boldsymbol{u}^{n+1 / 2} \cdot \nabla\right) \boldsymbol{u}^{n+1 / 2}$ can be treated in many ways. In Theorems 1 and 2, we use the Adams-Bashforth formula: $\frac{3}{2}\left(\boldsymbol{u}^{n} \cdot \nabla\right) \boldsymbol{u}^{n}-$ $\frac{1}{2}\left(\boldsymbol{u}^{n-1} \cdot \nabla\right) \boldsymbol{u}^{n-1}$. This explicit treatment of the convection term requires a CFL stability constraint. In the convergence analysis, this is realized by an a priori estimate (see (4.58)). This stability restriction and the a priori estimate are no longer needed in an implicit treatment of the convection term.

It is readily seen that the projection step enforces

$$
\frac{\partial p^{n+1}}{\partial \boldsymbol{n}}=\frac{\partial p^{n}}{\partial \boldsymbol{n}}=\cdots=\frac{\partial p^{0}}{\partial \boldsymbol{n}}=0, \quad \text { on } \partial \Omega,
$$

for the numerical solution. In general this is not satisfied by the exact solution of (2.1). Therefore we expect that $\partial p^{n} / \partial \boldsymbol{n}$ has $O(1)$ error at the boundary. This causes $\boldsymbol{u}^{*}$ and $p^{n}$ to have numerical boundary layers.

2.2. Spatial discretization. We will consider the case when the MAC scheme is used to discretize in $\boldsymbol{x}$. An illustration of the MAC mesh near the boundary is given in Figure 1. Here pressure is evaluated at the square points $(i, j)$, the $u$ velocity at the triangle points $(i \pm 1 / 2, j)$, and the $v$ velocity at the circle points $(i, j \pm 1 / 2)$. The discrete divergence is computed at the square points:

$$
(\nabla \cdot \boldsymbol{u})_{i, j}=\frac{u_{i+1 / 2, j}-u_{i-1 / 2, j}}{\triangle x}+\frac{v_{i, j+1 / 2}-v_{i, j-1 / 2}}{\triangle y} .
$$

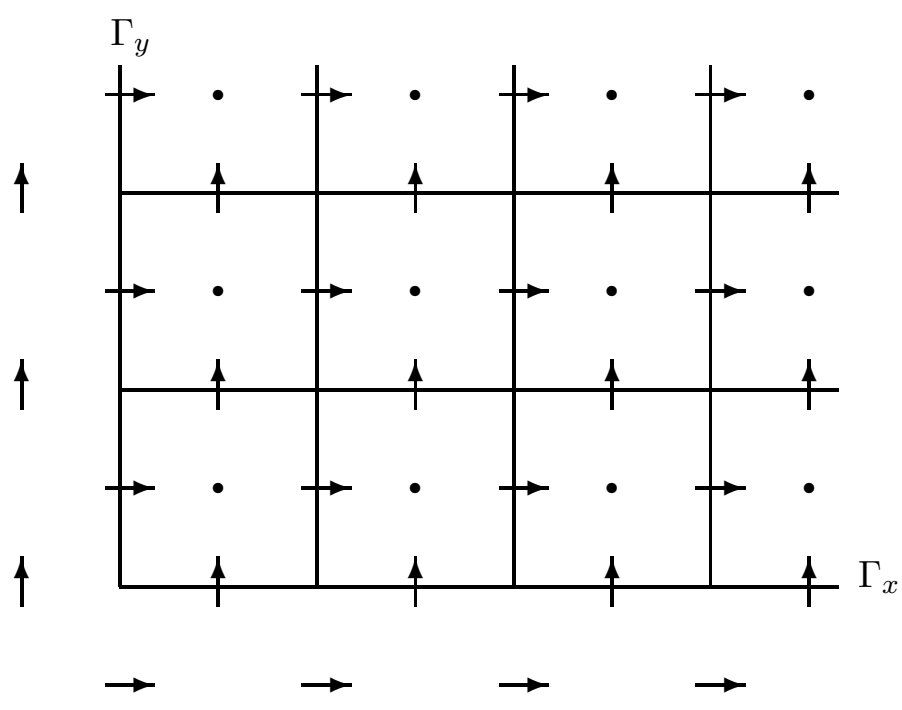

Figure 1. The MAC mesh 
Other differential operators are discretized as follows:

$$
\begin{aligned}
& (\Delta u)_{i+1 / 2, j}=\frac{u_{i+3 / 2, j}-2 u_{i+1 / 2, j}+u_{i-1 / 2, j}}{\triangle x^{2}} \\
& +\frac{u_{i+1 / 2, j+1}-2 u_{i+1 / 2, j}+u_{i+1 / 2, j-1}}{\triangle y^{2}} \\
& (\Delta v)_{i, j+1 / 2}=\frac{v_{i+1, j+1 / 2}-2 u_{i, j+1 / 2}+u_{i-1, j+1 / 2}}{\triangle x^{2}} \\
& +\frac{v_{i, j+3 / 2}-2 v_{i, j+1 / 2}+v_{i, j-1 / 2}}{\triangle y^{2}} \\
& \left(p_{x}\right)_{i+1 / 2, j}=\frac{p_{i+1, j}-p_{i, j}}{\triangle x} \\
& \left(p_{y}\right)_{i, j+1 / 2}=\frac{p_{i, j+1}-p_{i, j}}{\triangle y} \\
& \bar{u}_{i, j+1 / 2}=\frac{1}{4}\left(u_{i+1 / 2, j}+u_{i-1 / 2, j}+u_{i+1 / 2, j+1}+u_{i-1 / 2, j+1}\right) \\
& \bar{v}_{i+1 / 2, j}=\frac{1}{4}\left(v_{i+1, j+1 / 2}+v_{i+1, j-1 / 2}+v_{i, j+1 / 2}+v_{i, j-1 / 2}\right), \\
& \mathcal{N}_{h}(\boldsymbol{u}, a)_{i+1 / 2, j}=u_{i+1 / 2, j} \frac{a_{i+3 / 2, j}-a_{i-1 / 2, j}}{2 \triangle x}+\bar{v}_{i+1 / 2, j} \frac{a_{i+1 / 2, j+1}-a_{i+1 / 2, j-1}}{2 \triangle y}, \\
& \mathcal{N}_{h}(\boldsymbol{u}, b)_{i, j+1 / 2}=\bar{u}_{i, j+1 / 2} \frac{b_{i+1, j+1 / 2}-b_{i-1, j+1 / 2}}{2 \triangle x}+v_{i, j+1 / 2} \frac{b_{i, j+3 / 2}-b_{i, j-1 / 2}}{2 \triangle y},
\end{aligned}
$$

where we use the notation $\mathcal{N}$ to denote the nonlinear convection term. Clearly the truncation errors of these approximations are of second order.

The boundary condition $u=0$ is imposed at the vertical physical boundary, whereas $v=0$ is imposed at the "ghost" circle points which are $\frac{\Delta x}{2}$ to the left or right of the physical boundary. The "ghost" points are eliminated using linear interpolation of the boundary conditions. More explicitly, $v_{-1 / 2, j}+v_{1 / 2, j}=0$. Similarly the boundary condition $v=0$ is imposed at the horizontal physical boundary, but $u=0$ is imposed at the "ghost" triangle points a distance of $\frac{\Delta y}{2}$ away from the physical boundary.

One shortcoming of the MAC scheme is the serious constraint on geometry. Although slightly more general situations can be studied, in the present paper we will concentrate on the situation when $\Omega=[-1,1] \times[0,2 \pi]$ with periodic boundary condition in the $y$ direction and no-slip boundary condition in the $x$-direction: $\boldsymbol{u}(x, 0, t)=\boldsymbol{u}(x, 2 \pi, t), \boldsymbol{u}(-1, y, t)=0, \boldsymbol{u}(1, y, t)=0$. We will use $\partial^{\prime} \Omega$ to denote the part of the boundary at $x= \pm 1$. We will always assume that $\triangle x \sim \triangle y$ and $h=\min (\triangle x, \triangle y)$.

Notation. We will use $C$ to denote generic constants which may depend on the norms of the exact solutions. 


\section{SUMmaRY OF RESUlts}

The main results of this paper are the following (the constants are independent of $\triangle t$ and $h$ ).

Theorem 1. Let $(\boldsymbol{u}, p)$ be a solution of the Navier-Stokes equation (2.1) with smooth initial data $\boldsymbol{u}^{0}(\boldsymbol{x})$ satisfying the compatibility condition

$$
\boldsymbol{u}^{0}(\boldsymbol{x})=0, \quad \partial_{y} p(\boldsymbol{x}, 0)=\partial_{x y}^{2} p(\boldsymbol{x}, 0)=0, \quad \text { on } \partial^{\prime} \Omega .
$$

Let $\left(\boldsymbol{u}_{h}, p_{h}\right)$ be the numerical solution of the projection method (2.3)-(2.5) coupled with the MAC spatial discretization. Assume that $\triangle t<<$. Then we have

$$
\begin{gathered}
\left\|\boldsymbol{u}-\boldsymbol{u}_{h}\right\|_{L^{\infty}}+\triangle t^{1 / 2}\left\|p-p_{h}\right\|_{L^{\infty}} \leq C\left(\triangle t+h^{2}\right), \\
\left\|p-p_{h}-p_{c}\right\|_{L^{\infty}} \leq C\left(\triangle t+h^{2}\right),
\end{gathered}
$$

where

$$
\begin{array}{r}
p_{c}(\boldsymbol{x}, t) \equiv \Delta t^{1 / 2} \beta \frac{e^{\alpha}}{e^{\alpha}-1} e^{-\alpha|x-1| / \triangle t^{1 / 2}} D_{+}^{x} p_{h}\left(x-\triangle t^{1 / 2}, y, t\right) \\
+\triangle t^{1 / 2} \beta \frac{e^{\alpha}}{e^{\alpha}-1} e^{-\alpha|x+1| / \triangle t^{1 / 2}} D_{+}^{x} p_{h}\left(x+\triangle t^{1 / 2}, y, t\right), \\
\alpha=\frac{\triangle t^{1 / 2}}{\triangle x} \operatorname{arccosh}\left(1+\frac{\triangle x^{2}}{2 \triangle t}\right), \quad \beta=\frac{\triangle x}{\triangle t^{1 / 2}}\left(1-e^{-\alpha \triangle x / \triangle t^{1 / 2}}\right)^{-1} .
\end{array}
$$

Remark 1. As will be seen more clearly in the proof (4.52), at the scaling $\Delta=C h^{2}$ the boundary layer in pressure looks like $\Delta t^{1 / 2} A(y, t) \kappa^{j}$, where $A(y, t)$ is smooth, $|\kappa|<1, \kappa$ does not depend on $h$, and $j$ is the horizontal grid index relative to the lower boundary.

Theorem 2. Let $(\boldsymbol{u}, p)$ be a smooth solution of the Navier-Stokes equation (2.1) with smooth initial data $\boldsymbol{u}^{0}(\boldsymbol{x})$ satisfying the compatibility condition

$$
\partial_{x}^{\alpha_{1}} \partial_{y}^{\alpha_{2}} \boldsymbol{u}^{0}(\boldsymbol{x})=0, \quad \text { on } \partial \Omega, \text { for } \alpha_{1}+\alpha_{2} \leq 6 .
$$

Let $\left(\boldsymbol{u}_{h}, p_{h}\right)$ be the numerical solution of the projection method (2.6) coupled with the MAC spatial discretization. Assume that $\triangle t^{2}<<$. Then we have

$$
\begin{gathered}
\left\|\boldsymbol{u}-\boldsymbol{u}_{h}\right\|_{L^{\infty}}+\Delta t^{3 / 2}\left\|p-p_{h}\right\|_{L^{\infty}}+\triangle t\left\|p-p_{h}\right\|_{L^{\infty}\left(0, T, L^{2}\right)} \leq C\left(\Delta t^{2}+h^{2}\right), \\
\left\|p-p_{h}-p_{c}\right\|_{L^{\infty}} \leq C\left(\triangle t+h^{2}\right)
\end{gathered}
$$

where

$$
\begin{gathered}
p_{c} \equiv \Delta t^{1 / 2} \beta \frac{e^{\alpha}}{e^{\alpha}-1} e^{-\alpha|x-1| / \triangle t^{1 / 2}} D_{+}^{x} p_{h}\left(x-\triangle t^{1 / 2}, y, t\right) \\
+\triangle t^{1 / 2} \beta \frac{e^{\alpha}}{e^{\alpha}-1} e^{-\alpha|x+1| / \triangle t^{1 / 2}} D_{+}^{x} p_{h}\left(x+\triangle t^{1 / 2}, y, t\right), \\
\alpha=\frac{\triangle t^{1 / 2}}{\triangle x} \operatorname{arccosh}\left(1+\frac{\triangle x^{2}}{\triangle t}\right), \quad \beta=\frac{\triangle x}{\triangle t^{1 / 2}}\left(1-e^{-\alpha \Delta x / \triangle t^{1 / 2}}\right)^{-1} .
\end{gathered}
$$


Remark 2. The constraint on the size of $\Delta t$ is only technical and is different from the standard stability condition. The issue of the compatibility conditions on the initial data for the general domain are very complicated and are usually nonlocal [8]. In the case of periodic BC in $y$ and no-slip BC in $x$ (as in the present work), this reduces to the boundary condition (3.5). The correction term in (3.8) can also be given by $\frac{1}{2} \triangle t \Delta_{h} p$, as proposed in Kim \& Moin's original paper [9].

The proofs of these results follow the general strategy outlined in Section 3 of [3]. Step 3 is made simpler since we can now use the inverse inequality.

\section{First order schemes With SPATIAL Discretization}

We will concentrate on the following version of the first order projection method with the standard MAC spatial discretization:

$$
\left\{\begin{array}{l}
\frac{\boldsymbol{u}^{*}-\boldsymbol{u}^{n}}{\Delta t}+\mathcal{N}_{h}\left(\boldsymbol{u}^{n}, \boldsymbol{u}^{n}\right)=\Delta_{h} \boldsymbol{u}^{*} \\
\boldsymbol{u}^{*}=0, \quad \text { on } \partial^{\prime} \Omega \\
\boldsymbol{u}^{*}=\boldsymbol{u}^{n+1}+\triangle t \nabla_{h} p^{n} \\
\nabla_{h} \cdot \boldsymbol{u}^{n+1}=0, \\
\boldsymbol{n} \cdot \boldsymbol{u}^{n+1}=0, \quad \text { on } \partial^{\prime} \Omega
\end{array}\right.
$$

For $\boldsymbol{a}=(a, b), \boldsymbol{c}=(c, d), \boldsymbol{u}=(u, v)$, we define the following discrete inner products on the grid:

$$
\begin{aligned}
((\boldsymbol{a}, \boldsymbol{c}))= & \triangle x \triangle y \sum_{i=1}^{N-1} \sum_{j=1}^{N} a_{i+1 / 2, j} c_{i+1 / 2, j} \\
& +\triangle x \triangle y \sum_{i=1}^{N} \sum_{j=1}^{N} b_{i, j+1 / 2} d_{i, j+1 / 2} \\
\left(\left(\boldsymbol{u}, \nabla_{h} p\right)\right)= & \triangle y \sum_{i=1}^{N-1} \sum_{j=1}^{N} u_{i+1 / 2, j}\left(p_{i+1, j}-p_{i, j}\right) \\
& +\triangle x \sum_{i=1}^{N} \sum_{j=1}^{N} v_{i, j+1 / 2}\left(p_{i, j+1}-p_{i, j}\right) \\
\left(\left(\nabla_{h} \cdot \boldsymbol{u}, p\right)\right)= & \triangle y \sum_{i=1}^{N-1} \sum_{j=1}^{N}\left(u_{i+1 / 2, j}-u_{i-1 / 2, j}\right) p_{i, j} \\
& +\triangle x \sum_{i=1}^{N} \sum_{j=1}^{N}\left(v_{i, j+1 / 2}-v_{i, j-1 / 2}\right) p_{i, j},
\end{aligned}
$$

and discrete norms

$$
\|\boldsymbol{u}\|=((\boldsymbol{u}, \boldsymbol{u}))^{1 / 2}, \quad\|\boldsymbol{u}\|_{\infty}=\max _{i, j}\left|\boldsymbol{u}_{i, j}\right| .
$$

Denote $h=\min (\triangle x, \triangle y)$ 
Lemma 4.1. We have the following.

(i) Inverse inequality:

$$
\|f\|_{\infty} \leq \frac{1}{h}\|f\| .
$$

(ii) Poincaré inequality: if $\left.f\right|_{x= \pm 1}=0$, then

$$
\|f\| \leq C\left\|\nabla_{h} f\right\|
$$

where $C$ depends only on the domain.

(iii) Suppose $\left.\boldsymbol{n} \cdot \boldsymbol{u}\right|_{x= \pm 1}=0$; then we have

$$
\left(\left(\boldsymbol{u}, \nabla_{h} p\right)\right)=\left(\left(\nabla_{h} \cdot \boldsymbol{u}, p\right)\right) .
$$

(iv) Suppose $\left.\boldsymbol{u}\right|_{x= \pm 1}=0$; then we have

$$
2\left(\left(\boldsymbol{u}, \Delta_{h} \boldsymbol{u}\right)\right) \leq-\left\|\nabla_{h} \boldsymbol{u}\right\|^{2}-\left\|\nabla_{h} \cdot \boldsymbol{u}\right\|^{2} .
$$

(v) Suppose $\left.\boldsymbol{a}\right|_{x= \pm 1}=0$ and $\left.\boldsymbol{c} \cdot \boldsymbol{n}\right|_{\boldsymbol{x}= \pm 1}=0$; then we have

$$
\left|\left(\left(\boldsymbol{a}, \mathcal{N}_{h}(\boldsymbol{u}, \boldsymbol{c})\right)\right)\right| \leq\|\boldsymbol{c}\|\left\|\nabla_{h} \boldsymbol{a}\right\|\|\boldsymbol{u}\|_{W^{1, \infty}} .
$$

Proof. The proof of (i-iii) is standard. We show (iv). Summation by parts gives

$$
\begin{aligned}
\left(\left(\boldsymbol{u}, \Delta_{h} \boldsymbol{u}\right)\right)=-\left\|\nabla_{h} \boldsymbol{u}\right\|^{2}+\sum_{j} & {\left[v_{0, j+1 / 2}\left(v_{1, j+1 / 2}-v_{0, j+1 / 2}\right)\right.} \\
& \left.-v_{N, j+1 / 2}\left(v_{N+1, j+1 / 2}-v_{N, j+1 / 2}\right)\right] .
\end{aligned}
$$

Since $\left.v\right|_{x= \pm 1}=0$, we have

$$
v_{1, j+1 / 2}=-v_{0, j+1 / 2}, \quad v_{N, j+1 / 2}=-v_{N+1, j+1 / 2} .
$$

Hence

$$
\left(\left(\boldsymbol{u}, \Delta_{h} \boldsymbol{u}\right)\right)=-\left\|\nabla_{h} \boldsymbol{u}\right\|^{2}+2 \sum_{j}\left(v_{0, j+1 / 2}^{2}-v_{N, j+1 / 2}^{2}\right) .
$$

But

$$
\begin{aligned}
\left\|\nabla_{h} \boldsymbol{u}\right\|^{2} & \geq\left\|\nabla_{h} \cdot \boldsymbol{u}\right\|^{2}+\sum_{j}\left[\left(v_{1, j+1 / 2}-v_{0, j+1 / 2}\right)^{2}+\left(v_{N+1, j+1 / 2}-v_{N, j+1 / 2}\right)^{2}\right] \\
& =\left\|\nabla_{h} \cdot \boldsymbol{u}\right\|^{2}+4 \sum_{j}\left[\left(v_{0, j+1 / 2}\right)^{2}+\left(v_{N+1, j+1 / 2}\right)^{2}\right] .
\end{aligned}
$$

Combination of (4.11) and (4.12) gives (4.7).

To show (v), denote $I=\left(\left(\boldsymbol{a}, \mathcal{N}_{h}(\boldsymbol{u}, \boldsymbol{c})\right)\right)$. We have

$$
I=\triangle x \triangle y \sum_{i, j} a_{i+1 / 2, j}\left(u_{i+1 / 2, j} D_{0}^{x} c_{i+1 / 2, j}+\bar{v}_{i+1 / 2, j} D_{0}^{y} c_{i+1 / 2, j}\right)
$$

$$
+\triangle x \triangle y \sum_{i, j} b_{i, j+1 / 2}\left(\bar{u}_{i, j+1 / 2} D_{0}^{x} d_{i, j+1 / 2}+v_{i, j+1 / 2} D_{0}^{y} d_{i+1 / 2, j}\right)
$$


where we used the notations $\boldsymbol{a}=(a, b)$ and $\boldsymbol{c}=(c, d)$. Summation by parts gives $(4.14)$

$$
\begin{aligned}
I= & -\triangle x \triangle y \sum_{i, j} c_{i+1 / 2, j}\left[D_{0}^{x}\left(u_{i+1 / 2, j} a_{i+1 / 2, j}\right)+D_{0}^{y}\left(\bar{v}_{i+1 / 2, j} a_{i+1 / 2, j}\right)\right] \\
& -\triangle x \triangle y \sum_{i, j} d_{i, j+1 / 2}\left[D_{0}^{x}\left(\bar{u}_{i, j+1 / 2} b_{i, j+1 / 2}\right)+D_{0}^{y}\left(v_{i, j+1 / 2} b_{i, j+1 / 2}\right)\right] \\
& +\frac{1}{4} \triangle x \triangle y \sum_{j}\left(\bar{u}_{N+1, j+1 / 2} d_{N, j+1 / 2}-\bar{u}_{N, j+1 / 2} d_{N+1, j+1 / 2}\right) D_{-}^{x} b_{N+1, j+1 / 2} \\
& -\frac{1}{4} \triangle x \triangle y \sum_{j}\left(\bar{u}_{1, j+1 / 2} d_{0, j+1 / 2}-\bar{u}_{0, j+1 / 2} d_{1, j+1 / 2}\right) D_{+}^{x} b_{0, j+1 / 2} .
\end{aligned}
$$

Here we have used the fact that

$$
b_{1, j+1 / 2}=-b_{0, j+1 / 2}, \quad b_{N, j+1 / 2}=-b_{N+1, j+1 / 2} .
$$

Now, (4.8) follows directly. This completes the proof of the lemma.

We set $\varepsilon=\triangle t^{1 / 2}, \xi=(x+1) / \varepsilon, x_{i}=-1+i \triangle x, \xi_{i}=i \triangle \xi, \triangle \xi=\triangle x / \varepsilon$, $t^{n}=n \triangle t, t^{n-1 / 2}=(n-1 / 2) \triangle t, n=1,2, \cdots$. Clearly, we have

$$
\begin{aligned}
D_{+}^{\xi} \boldsymbol{a}\left(\xi_{i}, y_{j}, t\right) & =\frac{\boldsymbol{a}\left(\xi_{i+1}, y_{j}, t\right)-\boldsymbol{a}\left(\xi_{i}, y_{j}, t\right)}{\triangle \xi} \\
& =\varepsilon \frac{\boldsymbol{a}\left(x_{i+1} / \varepsilon, y_{j}, t\right)-\boldsymbol{a}\left(x_{i} / \varepsilon, y_{j}, t\right)}{\triangle x}=\varepsilon D_{+}^{x} \boldsymbol{a}\left(x_{i} / \varepsilon, y_{j}, t\right) .
\end{aligned}
$$

This shows that $D_{+}^{\xi}=\varepsilon D_{+}^{x}$. We will use the notation

$$
D_{\xi}^{2}=D_{-}^{\xi} D_{+}^{\xi}, \quad D_{y}^{2}=D_{-}^{y} D_{+}^{y},
$$

and

$$
\nabla_{\xi}=\left(D_{+}^{\xi}, 0\right), \quad \nabla_{y}=\left(0, D_{+}^{y}\right) .
$$

Denote the solutions of $(4.1)$ as $\left(\boldsymbol{u}_{h}, \boldsymbol{u}_{h}^{*}, p_{h}\right)$. Motivated by the discussions in $\S 4$ of [3], we make the following ansatz, valid at $t^{n}=n \triangle t, n=1,2, \cdots$ :

$$
\left\{\begin{array}{l}
\boldsymbol{u}_{h}^{*}(\boldsymbol{x}, t)=\boldsymbol{u}_{0}(\boldsymbol{x}, t)+\sum_{j=2} \varepsilon^{j}\left[\boldsymbol{u}_{j}^{*}(\boldsymbol{x}, t)+\boldsymbol{a}_{j}^{*}(\xi, y, t)\right] \\
\boldsymbol{u}_{h}(\boldsymbol{x}, t)=\boldsymbol{u}_{0}(\boldsymbol{x}, t)+\sum_{j=2} \varepsilon^{j} \boldsymbol{u}_{j}(\boldsymbol{x}, t) \\
p_{h}(\boldsymbol{x}, t)=p_{0}(\boldsymbol{x}, t)+\sum_{j=1} \varepsilon^{j}\left[p_{j}(\boldsymbol{x}, t)+\varphi_{j}(\xi, y, t)\right] .
\end{array}\right.
$$

Note that the functions involved are defined only on the numerical grid. So these formulas and the following ones should be understood as being valid on the grid. We have

$$
\Delta_{h} \boldsymbol{u}_{h}^{*}=\Delta_{h} \boldsymbol{u}_{0}+\sum_{j=2} \varepsilon^{j}\left(\Delta_{h} \boldsymbol{u}_{j}^{*}+\varepsilon^{-2} D_{\xi}^{2} \boldsymbol{a}_{j}^{*}+D_{y}^{2} \boldsymbol{a}_{j}^{*}\right)
$$




$$
\begin{gathered}
\nabla_{h} \cdot \boldsymbol{u}_{h}=\nabla_{h} \cdot \boldsymbol{u}_{0}+\sum_{j=2} \varepsilon^{j} \nabla_{h} \cdot \boldsymbol{u}_{j} \\
\nabla_{h} p_{h}=\nabla_{h} p_{0}+\varepsilon^{-1} \nabla_{\xi} \varphi_{0}+\nabla_{y} \varphi_{0}+\sum_{j=1} \varepsilon^{j}\left(\nabla_{h} p_{j}+\varepsilon^{-1} \nabla_{\xi} \varphi_{j}+\nabla_{y} \varphi_{j}\right) \\
\boldsymbol{u}_{h}^{n+1}(\boldsymbol{x})=\boldsymbol{u}_{0}\left(\boldsymbol{x}, t^{n+1}\right)+\sum_{j=2} \varepsilon^{j} \boldsymbol{u}_{j}\left(\boldsymbol{x}, t^{n+1}\right) \\
=\sum_{k=0} \frac{1}{k !} \varepsilon^{2 k} \boldsymbol{u}_{0}^{(k)}\left(\boldsymbol{x}, t^{n}\right)+\sum_{j=2} \varepsilon^{j} \sum_{k=0} \frac{1}{k !} \varepsilon^{2 k} \boldsymbol{u}_{j}^{(k)}\left(\boldsymbol{x}, t^{n}\right) .
\end{gathered}
$$

Next we substitute these relations into (4.1) in order to determine the coefficients of $\varepsilon^{j}$ in (4.19). We get hierarchies of equations by collecting equal powers of $\varepsilon$.

The first equation in (4.1) gives

$$
\boldsymbol{u}_{2}^{*}+\boldsymbol{a}_{2}^{*}-\boldsymbol{u}_{2}+\mathcal{N}_{h}\left(\boldsymbol{u}_{0}, \boldsymbol{u}_{0}\right)=\Delta_{h}^{2} \boldsymbol{u}_{0}^{*}+D_{\xi}^{2} \boldsymbol{a}_{2}^{*}
$$

For $j \geq 1$,

$$
\boldsymbol{u}_{j+2}^{*}+\boldsymbol{a}_{j+2}^{*}-\boldsymbol{u}_{j+2}+\sum_{k=0}^{j} \mathcal{N}_{h}\left(\boldsymbol{u}_{k}, \boldsymbol{u}_{j-k}\right)=\Delta_{h} \boldsymbol{u}_{j}^{*}+D_{\xi}^{2} \boldsymbol{a}_{j+2}^{*}+D_{y}^{2} \boldsymbol{a}_{j}^{*} .
$$

The second equation in (4.1) implies that

$$
\boldsymbol{u}_{2}^{*}+\boldsymbol{a}_{2}^{*}=\boldsymbol{u}_{2}+\partial_{t} \boldsymbol{u}_{0}+\nabla_{h} p_{0}+\nabla_{\xi} \varphi_{1}+\nabla_{y} \varphi_{0}
$$

For $j=2 \ell-1, \ell \geq 1$,

$$
\boldsymbol{u}_{j+2}^{*}+\boldsymbol{a}_{j+2}^{*}=\boldsymbol{u}_{j+2}+\partial_{t} \boldsymbol{u}_{j}+\nabla_{h} p_{j}+\nabla_{\xi} \varphi_{j+1}+\nabla_{y} \varphi_{j}+\sum_{k=2}^{\ell} \frac{1}{k !} \boldsymbol{u}_{j-2 k+2}^{(k)}
$$

For $j=2 \ell, \ell \geq 1$,

$$
\begin{aligned}
\boldsymbol{u}_{j+2}^{*}+\boldsymbol{a}_{j+2}^{*}= & \boldsymbol{u}_{j+2}+\partial_{t} \boldsymbol{u}_{j}+\nabla_{h} p_{j}+\nabla_{\xi} \varphi_{j+1}+\nabla_{y} \varphi_{j} \\
& +\frac{1}{(\ell+1) !} \boldsymbol{u}_{0}^{(\ell+1)}+\sum_{k=2}^{\ell} \frac{1}{k !} \boldsymbol{u}_{j-2 k+2}^{(k)} .
\end{aligned}
$$

From the third equation in (4.1), we obtain

$$
\nabla_{h} \cdot \boldsymbol{u}_{j}=0, \quad j=0,1, \cdots
$$

The boundary conditions become

$$
\boldsymbol{u}_{j}^{*}+\boldsymbol{a}_{j}^{*}=0, \quad D_{+}^{x} p_{j-1}+D_{+}^{\xi} \varphi_{j}=0, \quad \text { at } \quad x=-1, \quad \xi=0,
$$

for $j>0$.

Next we go through all these equations, one by one, to see if they are solvable. Since this is very similar to $\S 4.1$ in [3], we will only give a summary of results.

The coefficients in the expansions (4.19) can be obtained successively in the following order:

$$
\left\{\begin{array}{l}
\partial_{t} \boldsymbol{u}_{0}+\nabla_{h} p_{0}+\mathcal{N}_{h}\left(\boldsymbol{u}_{0}, \boldsymbol{u}_{0}\right)=\Delta_{h} \boldsymbol{u}_{0} \\
\nabla_{h} \cdot \boldsymbol{u}_{0}=0, \\
\boldsymbol{u}_{0}=0, \text { at } \quad x= \pm 1 \\
\boldsymbol{u}_{0}(\cdot, 0)=\boldsymbol{u}^{0}(\cdot)
\end{array}\right.
$$


Using the following lemma, we know that (4.31) has a smooth solution in the sense that the divided differences of various orders are bounded.

Lemma 4.2. Let $(\boldsymbol{u}, p)$ be a solution of the Navier-Stokes equation (2.1) with smooth initial data $\boldsymbol{u}^{0}(\boldsymbol{x})$ satisfying some compatibility conditions. Let $\left(\boldsymbol{u}_{0}, p_{0}\right)$ be a solution of (4.31). Then $\left(\boldsymbol{u}_{0}, p_{0}\right)$ is smooth in the sense that its discrete derivatives are bounded. Moreover, we have

$$
\left\|\boldsymbol{u}-\boldsymbol{u}_{0}\right\|_{L^{\infty}}+\left\|p-p_{0}\right\|_{L^{\infty}} \leq C h^{2} .
$$

The proof of this lemma, as well as Lemma 4.3, can be found in [15].

We next have

$$
\begin{gathered}
\boldsymbol{u}_{2}^{*}=\boldsymbol{u}_{2}+\partial_{t} \boldsymbol{u}_{0}+\nabla_{h} p_{0}, \\
\left\{\begin{array}{l}
\varphi_{1}=D_{\xi}^{2} \varphi_{1}, \\
\left.D_{+}^{\xi} \varphi_{1}\right|_{\xi=0}=-\left.D_{+}^{x} p_{0}\right|_{x=-1} .
\end{array}\right.
\end{gathered}
$$

This gives

$$
\varphi_{1}(\xi, y, t)=\beta D_{+}^{x} p_{0}(-1, y, t) e^{-\alpha \xi}
$$

where

$$
\begin{gathered}
\alpha=\frac{1}{\triangle \xi} \operatorname{arccosh}\left(1+\Delta \xi^{2} / 2\right), \quad \beta=\Delta \xi\left(1-e^{-\alpha \Delta \xi}\right)^{-1}, \\
a_{2}^{*}=D_{+}^{\xi} \varphi_{1}, \quad b_{2}^{*}=0 \\
\boldsymbol{u}_{3}^{*}=\boldsymbol{u}_{3}, \\
\varphi_{2}=0, \quad a_{3}^{*}=0, \quad b_{3}^{*}=D_{+}^{y} \varphi_{1}, \\
\left\{\begin{array}{c}
\partial_{t} \boldsymbol{u}_{2}+\nabla_{h} p_{2}+\mathcal{N}_{h}\left(\boldsymbol{u}_{0}, \boldsymbol{u}_{2}\right)+\mathcal{N}_{h}\left(\boldsymbol{u}_{2}, \boldsymbol{u}_{0}\right) \\
=\Delta_{h} \boldsymbol{u}_{2}+\Delta_{h}\left(\partial_{t} \boldsymbol{u}_{0}+\nabla_{h} p_{0}\right)-\frac{1}{2} \partial_{t}^{2} \boldsymbol{u}_{0}, \\
\nabla_{h} \cdot \boldsymbol{u}_{2}=0, \\
\left.\boldsymbol{u}_{2}\right|_{x=-1}=-\left.\nabla_{h} p_{0}\right|_{x=-1}-\left.\nabla_{\xi} \varphi_{1}\right|_{\xi=0}, \quad \text { on } \partial \Omega .
\end{array}\right.
\end{gathered}
$$

With suitable initial data, we know from the following lemma that (4.40) has a smooth solution.

Lemma 4.3. Let $(\boldsymbol{u}, p)$ be a solution of the linear $O D E$

$$
\left\{\begin{array}{l}
\partial_{t} \boldsymbol{u}+\nabla_{h} p+\mathcal{N}_{h}\left(\boldsymbol{u}_{0}, \boldsymbol{u}\right)+\mathcal{N}_{h}\left(\boldsymbol{u}, \boldsymbol{u}_{0}\right)=\Delta_{h} \boldsymbol{u}+\boldsymbol{f} \\
\nabla_{h} \cdot \boldsymbol{u}=0, \\
\boldsymbol{u}=\boldsymbol{g}, \quad \text { at } \quad x= \pm 1 \\
\boldsymbol{u}(\cdot, 0)=\boldsymbol{u}^{0}(\cdot)
\end{array}\right.
$$

where $\boldsymbol{f}, \boldsymbol{g}$ and $\boldsymbol{u}^{0}$ smooth and satisfy some compatibility conditions. Then $(\boldsymbol{u}, p)$ is smooth in the sense that its divided differences of various order are bounded.

Continuing in this fashion, we get

$$
\left\{\begin{array}{l}
\varphi_{3}=D_{\xi}^{2} \varphi_{3}+D_{y}^{2} \varphi_{1} \\
\left.D_{+}^{\xi} \varphi_{3}\right|_{\xi=0}=-\left.D_{+}^{x} p_{2}\right|_{x=-1} .
\end{array}\right.
$$

The solution for $(4.42)$ is

$$
\varphi_{3}(\xi, y, t)=\beta D_{+}^{x} p_{2} e^{-\alpha \xi}+\left.\beta_{1}(\xi+\gamma) D_{+}^{x} D_{y}^{2} p_{0}\right|_{x=-1} e^{-\alpha \xi},
$$


where

$$
\begin{gathered}
\beta_{1}=\frac{1}{\left(1-e^{-\alpha \triangle \xi}\right)\left(e^{-\alpha \triangle \xi}-e^{\alpha \triangle \xi}\right)}, \quad \gamma=\Delta \xi \frac{e^{-\alpha \triangle \xi}}{1-e^{-\alpha \triangle \xi}}, \\
b_{4}^{*}=0, \quad a_{4}^{*}=D_{+}^{\xi} \varphi_{3} . \\
\left\{\begin{array}{l}
\partial_{t} \boldsymbol{u}_{3}+\nabla_{h} p_{3}+\mathcal{N}_{h}\left(\boldsymbol{u}_{0}, \boldsymbol{u}_{3}\right)+\mathcal{N}_{h}\left(\boldsymbol{u}_{3}, \boldsymbol{u}_{0}\right)=\Delta_{h} \boldsymbol{u}_{3}+\Delta_{h} \nabla_{h} p_{2}, \\
\nabla_{h} \cdot \boldsymbol{u}_{3}=0, \\
\left.\boldsymbol{u}_{3}\right|_{x=-1}=-\left.\nabla_{y} \varphi_{1}\right|_{\xi=0}, \\
\varphi_{4}=D_{\xi}^{2} \varphi_{4}, \\
\left.D_{+}^{\xi} \varphi_{4}\right|_{\xi=0}=-\left.D_{+}^{x} p_{3}\right|_{x=-1}, \\
a_{5}^{*}=D_{+}^{\xi} \varphi_{4}, \quad b_{5}^{*}=D_{+}^{y} \varphi_{3} .
\end{array}\right.
\end{gathered}
$$

Obviously this procedure can be continued, and we obtain

$$
\begin{aligned}
& \left\{\begin{array}{l}
\varphi_{j}=D_{\xi}^{2} \varphi_{j}+D_{y}^{2} \varphi_{j-2}, \\
\left.D_{+}^{\xi} \varphi_{j}\right|_{\xi=0}=-\left.D_{+}^{x} p_{j-1}\right|_{x=-1},
\end{array}\right. \\
& \varphi_{j}=\sum_{k=0}^{[j / 2]} F_{j, k}(y) \xi^{k} e^{-\alpha \xi} \\
& a_{j}^{*}=D_{+}^{\xi} \varphi_{j-1}, \quad b_{j}^{*}=D_{+}^{y} \varphi_{j-2},
\end{aligned}
$$

Now if we let

$$
\left\{\begin{array}{l}
U^{*}=\boldsymbol{u}_{0}^{*}+\sum_{j=1}^{2 N} \varepsilon^{j}\left(\boldsymbol{u}_{j}^{*}+\boldsymbol{a}_{j}^{*}\right) \\
U^{n}=\boldsymbol{u}_{0}+\sum_{j=1}^{2 N} \varepsilon^{j} \boldsymbol{u}_{j} \\
P^{n}=p_{0}+\sum_{j=1}^{2 N} \varepsilon^{j}\left(p_{j}+\varphi_{j}\right)+\varepsilon^{2 N+1} \varphi_{2 N+1}
\end{array}\right.
$$

then we have

$$
\left\{\begin{array}{l}
\frac{U^{*}-U^{n}}{\triangle t}+\mathcal{N}_{h}\left(U^{n}, U^{n}\right)=\Delta_{h} U^{*}+\Delta t^{\alpha} \boldsymbol{f} \\
U^{*}=0, \quad \text { at } \quad x= \pm 1 \\
U^{*}=U^{n+1}+\triangle t \nabla_{h} P^{n}+\Delta t^{\alpha+1} \boldsymbol{g} \\
\nabla_{h} \cdot U^{n+1}=0, \\
D_{+}^{x} P^{n}=\boldsymbol{n} \cdot U^{n+1}=0, \quad \text { at } \quad x= \pm 1
\end{array}\right.
$$

where $\alpha=N-1 / 2 ; \boldsymbol{f}$ and $\boldsymbol{g}$ are bounded and smooth if $\left(\boldsymbol{u}_{0}, p_{0}\right)$ is sufficiently smooth. It is easy to see that

$$
\max _{0 \leq t \leq T}\left\|U^{n}(\cdot)\right\|_{W^{1, \infty}} \leq C^{*} .
$$


We point out that the constructed solution in (4.52) is different from the discretized solution. The constructed solution consists of two parts: the leading term is the exact solution of the NSE on MAC grid (4.31) and hence is bounded (see [15]), and the remaining terms are the solutions of the linearized (linearized on the exact solution) NSE (4.40), (4.46), and hence are bounded. As a direct consequence, the truncation terms $\boldsymbol{f}$ and $\boldsymbol{g}$ in (4.53) are also bounded.

For the initial data, we have

$$
U^{0}(\boldsymbol{x})=\boldsymbol{u}^{0}(\boldsymbol{x})+\triangle t \boldsymbol{w}^{0}(\boldsymbol{x})
$$

where $\boldsymbol{w}^{0}$ is a bounded function. Furthermore, under the compatibility condition (3.5), we can construct a better approximate initial data

$$
U^{0}(\boldsymbol{x})=\boldsymbol{u}^{0}(\boldsymbol{x})+\triangle t^{2} \boldsymbol{w}^{0}(\boldsymbol{x}) .
$$

The construction of the initial $\boldsymbol{w}^{0}$ in (4.55) and (4.57) in general is very complicated. This is exactly where we need the compatibility conditions. In the case of periodic BC for $y$ and no-slip boundary condition in $x$ (the domain we considered in this paper), the construction of $\boldsymbol{w}^{0}$ is more straightforward. The details of the construction are very similar to those used in the analysis of the gauge method in [14. In that paper the details of the construction of $\boldsymbol{w}^{0}$ were given.

Proof of Theorem 1. Assume a priori that

$$
\max _{0 \leq t^{n} \leq T}\left\|\boldsymbol{u}^{n}\right\|_{W^{1, \infty}} \leq \tilde{C} .
$$

We will justify this a priori assumption later (see (4.67)).

In the following estimates, the constant will sometimes depend on $C^{*}$ and $\tilde{C}$, and we will show that it is uniformly bounded. Later on we will estimate $\tilde{C}$. Let

$$
\boldsymbol{e}^{n}=U^{n}-\boldsymbol{u}^{n}, \quad \boldsymbol{e}^{*}=U^{*}-\boldsymbol{u}^{*}, \quad q^{n}=P^{n}-p^{n} .
$$

Subtracting (4.53) from (4.1), we get the following error equation:

$$
\left\{\begin{array}{l}
\frac{\boldsymbol{e}^{*}-\boldsymbol{e}^{n}}{\triangle t}+\mathcal{N}_{h}\left(\boldsymbol{e}^{n}, U^{n}\right)+\mathcal{N}_{h}\left(\boldsymbol{u}^{n}, \boldsymbol{e}^{n}\right)=\Delta_{h} \boldsymbol{e}^{*}+\triangle t^{\alpha} \boldsymbol{f}^{n}, \\
\boldsymbol{e}^{*}=0, \quad \text { at } \quad x= \pm 1 \\
\frac{\boldsymbol{e}^{n+1}-\boldsymbol{e}^{*}}{\triangle t}+\nabla_{h} q^{n}=\Delta t^{\alpha} \boldsymbol{g}^{n}, \\
\nabla_{h} \cdot \boldsymbol{e}^{n+1}=0, \\
D_{+}^{x} q^{n}=\boldsymbol{e}^{n+1} \cdot \boldsymbol{n}=0, \quad \text { at } \quad x= \pm 1 \\
\boldsymbol{e}^{0}=\Delta t^{\alpha} \boldsymbol{w}^{0} .
\end{array}\right.
$$

Taking the scalar product of the first equation of (4.60) with $2 e^{*}$ and integrating by parts, we obtain

$$
\begin{aligned}
\left\|\boldsymbol{e}^{*}\right\|^{2} & -\left\|\boldsymbol{e}^{n}\right\|^{2}+\left\|\boldsymbol{e}^{*}-\boldsymbol{e}^{n}\right\|^{2}+\triangle t\left\|\nabla_{h} \boldsymbol{e}^{*}\right\|^{2} \\
\leq & \triangle t^{2 \alpha+1}\left\|\boldsymbol{f}^{n}\right\|^{2}+\triangle t\left\|\boldsymbol{e}^{*}\right\|^{2}-2 \triangle t\left(\left(\boldsymbol{e}^{*}, \mathcal{N}_{h}\left(\boldsymbol{e}^{n}, U^{n}\right)\right)\right) \\
& -2 \triangle t\left(\left(\boldsymbol{e}^{*}, \mathcal{N}_{h}\left(\boldsymbol{u}^{n}, \boldsymbol{e}^{n}\right)\right)\right) \\
\leq & \triangle t^{2 \alpha+1}\left\|\boldsymbol{f}^{n}\right\|^{2}+C \triangle t\left(\left\|\boldsymbol{e}^{*}\right\|^{2}+\left\|\boldsymbol{e}^{n}\right\|^{2}\right)+\frac{1}{2} \triangle t\left\|\nabla_{h} \boldsymbol{e}^{*}\right\|^{2} .
\end{aligned}
$$


Here we used Lemma 4.1. Taking the scalar product of the second equation of (4.60) with $2 e^{n+1}$ yields

$$
\left\|e^{n+1}\right\|^{2}-\left\|e^{*}\right\|^{2}+\left\|e^{n+1}-e^{*}\right\|^{2} \leq \Delta t\left\|e^{n+1}\right\|^{2}+\Delta t^{2 \alpha+1}\left\|\boldsymbol{g}^{n}\right\|^{2} .
$$

Combining (4.61) and (4.62), we get

$$
\begin{gathered}
\left\|e^{n+1}\right\|^{2}-\left\|e^{n}\right\|^{2}+\left\|e^{*}-e^{n}\right\|^{2}+\left\|e^{n+1}-e^{*}\right\|^{2}+\triangle t\left\|\nabla_{h} e^{*}\right\|^{2} \\
\leq C \triangle t\left(\left\|e^{n+1}\right\|^{2}+\left\|e^{n}\right\|^{2}\right)+\triangle t^{2 \alpha+1}\left(\left\|\boldsymbol{f}^{n}\right\|^{2}+\left\|\boldsymbol{g}^{n}\right\|^{2}\right) .
\end{gathered}
$$

Applying the discrete Gronwall lemma to the last inequality, we arrive at

$$
\left\|e^{n}\right\|+\left\|e^{*}-e^{n}\right\|+\left\|e^{n+1}-e^{*}\right\|+\triangle t^{1 / 2}\left\|\nabla_{h} e^{*}\right\| \leq C_{1} \triangle t^{\alpha} .
$$

Using the second equation of (4.60), we have

$$
\left\|e^{n}\right\|+\triangle t\left\|\nabla_{h} q^{n}\right\| \leq C_{1} \triangle t^{\alpha} .
$$

Now by the inverse inequality (4.4) we have

$$
\left\|\boldsymbol{e}^{n}\right\|_{L^{\infty}}+\triangle t\left\|\nabla_{h} q^{n}\right\|_{L^{\infty}}+h\left\|\boldsymbol{e}^{n}\right\|_{W^{1, \infty}} \leq C_{1} \frac{\triangle t^{\alpha}}{h} .
$$

If $N=3$ and $\triangle t^{\alpha}<<h^{2}$, and we choose $\triangle t$ small enough, we will always have

$$
\left\|e^{n+1}\right\|_{W^{1, \infty}} \leq 1 \text {. }
$$

Therefore in (4.58) we can choose

$$
\tilde{C}=1+\max _{n \leq\left[\frac{T}{\Delta t}\right]+1}\left\|U^{n}(\cdot)\right\|_{W^{1, \infty}},
$$

which depends only on the exact solution $(\boldsymbol{u}, p)$. This proves that

$$
\left\|\boldsymbol{u}_{0}-\boldsymbol{u}_{h}\right\|_{L^{\infty}}+\left\|p_{0}-p_{h}\right\|_{L^{2}}+\triangle t^{1 / 2}\left\|p_{0}-p_{h}\right\|_{L^{\infty}}+\left\|p_{0}-p_{h}-p_{c}\right\|_{L^{\infty}} \leq C \triangle t .
$$

But we also have, from Lemma 4.2,

$$
\left\|\boldsymbol{u}-\boldsymbol{u}_{0}\right\|_{L^{\infty}}+\left\|p-p_{0}\right\|_{L^{\infty}} \leq C h^{2} .
$$

Thus

$$
\left\|\boldsymbol{u}-\boldsymbol{u}_{h}\right\|_{L^{\infty}}+\left\|p-p_{h}\right\|_{L^{2}}+\triangle t^{1 / 2}\left\|p-p_{h}\right\|_{L^{\infty}}+\left\|p-p_{h}-p_{c}\right\|_{L^{\infty}} \leq C\left(\triangle t+h^{2}\right) .
$$

This completes the proof of Theorem 1 .

\section{SECOND ORDER SCHEMES With SPATIAL DiscRETIZATION}

In this section we carry out the same program as in $\S 4$ for the second order method (2.6) with the standard MAC spatial discretization:

$$
\left\{\begin{array}{l}
\frac{\boldsymbol{u}^{*}-\boldsymbol{u}^{n}}{\Delta t}=\Delta_{h} \frac{\boldsymbol{u}^{*}+\boldsymbol{u}^{n}}{2}, \\
\boldsymbol{u}^{*}+\boldsymbol{u}^{n}=\triangle t \nabla_{h} p^{n-1 / 2}, \quad \text { at } \quad x= \pm 1 \\
\boldsymbol{u}^{*}=\boldsymbol{u}^{n+1}+\triangle t \nabla_{h} p^{n+1 / 2}, \\
\nabla_{h} \cdot \boldsymbol{u}^{n+1}=0, \\
\boldsymbol{n} \cdot \boldsymbol{u}^{n+1}=0, \quad \text { at } \quad x= \pm 1 .
\end{array}\right.
$$

Here we leave out the nonlinear term, since it does not affect the major steps but substantially complicates the presentation. 
We begin with the following ansatz:

$$
\left\{\begin{array}{l}
\boldsymbol{u}^{*}(\boldsymbol{x})=\boldsymbol{u}_{0}\left(\boldsymbol{x}, t^{n}\right)+\sum_{j=2} \varepsilon^{j}\left[\boldsymbol{u}_{j}^{*}\left(\boldsymbol{x}, t^{n}\right)+\boldsymbol{a}_{j}^{*}\left(\xi, y, t^{n}\right)\right] \\
\boldsymbol{u}^{n}(\boldsymbol{x})=\boldsymbol{u}_{0}\left(\boldsymbol{x}, t^{n}\right)+\sum_{j=4} \varepsilon^{j} \boldsymbol{u}_{j}\left(\boldsymbol{x}, t^{n}\right) \\
p^{n-1 / 2}(\boldsymbol{x})=p_{0}\left(\boldsymbol{x}, t^{n-1 / 2}\right)+\varepsilon \varphi_{1}\left(\xi, y, t^{n-1 / 2}\right)+\varepsilon^{3} \varphi_{3}\left(\xi, y, t^{n-1 / 2}\right) \\
+\sum_{j=4} \varepsilon^{j}\left[p_{j}\left(\boldsymbol{x}, t^{n-1 / 2}\right)+\varphi_{j}\left(\xi, y, t^{n-1 / 2}\right)\right]
\end{array}\right.
$$

Here again we set $\varepsilon=\Delta t^{1 / 2}, \xi=(x+1) / \varepsilon, t^{n}=n \triangle t, t^{n-1 / 2}=(n-1 / 2) \triangle t$, $n=1,2, \cdots$. The formulas are to be understood as being valid at the grid points. Substituting (5.2) into (5.1) and collecting equal powers of $\varepsilon$, we get the following equations:

From the first equation in (5.1), we get

$$
\boldsymbol{u}_{2}^{*}+\boldsymbol{a}_{2}^{*}-\boldsymbol{u}_{2}=\frac{1}{2}\left(\Delta_{h} \boldsymbol{u}_{0}^{*}+D_{\xi}^{2} \boldsymbol{a}_{2}^{*}+\Delta_{h} \boldsymbol{u}_{0}\right)
$$

For $j \geq 1$,

$$
\boldsymbol{u}_{j+2}^{*}+\boldsymbol{a}_{j+2}^{*}-\boldsymbol{u}_{j+2}=\frac{1}{2}\left(\Delta_{h} \boldsymbol{u}_{j}^{*}+D_{\xi}^{2} \boldsymbol{a}_{j+2}^{*}+D_{y}^{2} \boldsymbol{a}_{j}^{*}+\Delta_{h} \boldsymbol{u}_{j}\right) .
$$

From the third equation in (5.1), we get

$$
\begin{gathered}
\boldsymbol{u}_{2}^{*}+\boldsymbol{a}_{2}^{*}=\boldsymbol{u}_{2}+\partial_{t} \boldsymbol{u}_{0}+\nabla_{h} p_{0}+\nabla_{\xi} \varphi_{1}, \\
\boldsymbol{u}_{3}^{*}+\boldsymbol{a}_{3}^{*}=\boldsymbol{u}_{3}+\partial_{t} \boldsymbol{u}_{1}+\nabla_{h} p_{1}+\nabla_{\xi} \varphi_{2}+\nabla_{y} \varphi_{1} .
\end{gathered}
$$

For $j=2 \ell$,

$$
\begin{aligned}
\boldsymbol{u}_{j+2}^{*}+ & \boldsymbol{a}_{j+2}^{*} \\
= & \boldsymbol{u}_{j+2}+\partial_{t} \boldsymbol{u}_{j}+\nabla_{h} p_{j}+\nabla_{\xi} \varphi_{j+1}+\nabla_{y} \varphi_{j} \\
& +\frac{1}{(\ell+1) !} \boldsymbol{u}_{0}^{(\ell+1)}+\sum_{k=2}^{\ell} \frac{1}{k !} \boldsymbol{u}_{j-2 k+2}^{(k)}+\frac{1}{2^{\ell} \ell !} \nabla_{h} p_{0}^{(\ell)}+\sum_{k=1}^{\ell-1} \frac{1}{2^{k} k !} \nabla_{h} p_{j-2 k}^{(k)} \\
& +\sum_{k=1}^{\ell} \frac{1}{2^{k} k !}\left(\nabla_{\xi} \varphi_{j-2 k+1}^{(k)}+\nabla_{y} \varphi_{j-2 k}^{(k)}\right)
\end{aligned}
$$

For $j=2 \ell+1$,

$$
\begin{aligned}
\boldsymbol{u}_{j+2}^{*} & +\boldsymbol{a}_{j+2}^{*} \\
& =\boldsymbol{u}_{j+2}+\partial_{t} \boldsymbol{u}_{j}+\nabla_{h} p_{j}+\nabla_{\xi} \varphi_{j+1}+\nabla_{y} \varphi_{j} \\
& +\sum_{k=2}^{\ell+1} \frac{1}{k !} \boldsymbol{u}_{j-2 k+2}^{(k)}+\sum_{k=1}^{\ell} \frac{1}{2^{k} k !}\left(\nabla_{h} p_{j-2 k}^{(k)}+\nabla_{\xi} \varphi_{j-2 k+1}^{(k)}+\nabla_{y} \varphi_{j-2 k}^{(k)}\right) .
\end{aligned}
$$

From the incompressibility condition, we get

$$
\nabla_{h} \cdot \boldsymbol{u}_{j}=0, \text { for } j \geq 0 .
$$


The boundary conditions imply that for $x=-1, \xi=0$,

$$
\begin{gathered}
\boldsymbol{u}_{0}=0 \\
\boldsymbol{u}_{2}+\boldsymbol{u}_{2}^{*}+\boldsymbol{a}_{2}^{*}=\nabla_{h} p_{0}+\nabla_{\xi} \varphi_{1}, \\
\boldsymbol{u}_{3}+\boldsymbol{u}_{3}^{*}+\boldsymbol{a}_{3}^{*}=\nabla_{h} p_{1}+\nabla_{\xi} \varphi_{2}+\nabla_{y} \varphi_{1} ;
\end{gathered}
$$

for $j=2 \ell, \ell \geq 1$,

$$
\begin{aligned}
\boldsymbol{u}_{j}+ & \boldsymbol{u}_{j}^{*}+\boldsymbol{a}_{j}^{*} \\
= & \nabla_{h} p_{j-2}+\nabla_{\xi} \varphi_{j-1}+\nabla_{y} \varphi_{j-2}+\frac{(-1)^{\ell-1}}{2^{\ell-1}} \frac{1}{(\ell-1) !} \nabla_{h} p_{0}^{(\ell-1)} \\
& +\sum_{k=1}^{\ell-2} \frac{(-1)^{k}}{2^{k} k !} \nabla_{h} p_{j-2 k-2}^{(k)}+\sum_{k=1}^{\ell-1} \frac{(-1)^{k}}{2^{k} k !}\left(\nabla_{\xi} \varphi_{j-2 k-1}^{(k)}+\nabla_{y} \varphi_{j-2 k-2}^{(k)}\right) ;
\end{aligned}
$$

for $j=2 \ell+1, \ell \geq 1$,

$$
\begin{aligned}
\boldsymbol{u}_{j}+\boldsymbol{u}_{j}^{*}+\boldsymbol{a}_{j}^{*}= & \nabla_{h} p_{j-2}+\nabla_{\xi} \varphi_{j-1}+\nabla_{y} \varphi_{j-2} \\
& +\sum_{k=1}^{\ell-1} \frac{(-1)^{k}}{2^{k} k !}\left(\nabla_{h} p_{j-2 k-2}^{(k)}+\nabla_{\xi} \varphi_{j-2 k-1}^{(k)}+\nabla_{y} \varphi_{j-2 k-2}^{(k)}\right) ;
\end{aligned}
$$

and for $j \geq 0$

$$
D_{+}^{x} p_{j}+D_{+}^{\xi} \varphi_{j+1}=0 .
$$

Next we go through all these equations, one by one, to see if they are solvable. It can be checked that the coefficients in the expansions (5.2) can be obtained successively in the following order:

$$
\begin{gathered}
\left\{\begin{array}{l}
\partial_{t} \boldsymbol{u}_{0}+\nabla_{h} p_{0}=\Delta_{h} \boldsymbol{u}_{0}, \\
\nabla_{h} \cdot \boldsymbol{u}_{0}=0, \\
\boldsymbol{u}_{0}=0, \quad \text { at } \quad x= \pm 1,
\end{array}\right. \\
\boldsymbol{u}_{2}^{*}=\boldsymbol{u}_{2}+\partial_{t} \boldsymbol{u}_{0}+\nabla_{h} p_{0}, \\
\left\{\begin{array}{l}
\varphi_{1}=\frac{1}{2} D_{\xi}^{2} \varphi_{1}, \\
\left.D_{+}^{\xi} \varphi_{1}\right|_{\xi=0}=-\left.D_{+}^{x} p_{0}\right|_{x=-1},
\end{array}\right. \\
\varphi_{1}=\left.\beta D_{+}^{x} p_{0}\right|_{x=-1} e^{-\alpha \xi},
\end{gathered}
$$

where

$$
\begin{gathered}
\alpha=\frac{1}{\triangle \xi} \operatorname{arccosh}\left(1+\triangle \xi^{2}\right), \quad \beta=\triangle \xi\left(1-e^{-\alpha \triangle \xi}\right)^{-1}, \\
a_{2}^{*}=D_{+}^{\xi} \varphi_{1}, \quad b_{2}^{*}=0 .
\end{gathered}
$$


We next have

$$
\begin{gathered}
\boldsymbol{u}_{3}^{*}=\boldsymbol{u}_{3}, \\
\varphi_{2}=0, \quad a_{3}^{*}=0, \quad b_{3}^{*}=D_{+}^{y} \varphi_{1}, \\
\left\{\begin{array}{l}
\varphi_{3}=\frac{1}{2}\left(D_{\xi}^{2} \varphi_{3}+D_{y}^{2} \varphi_{1}\right), \\
\left.D_{+}^{\xi} \varphi_{3}\right|_{\xi=0}=0 .
\end{array}\right.
\end{gathered}
$$

The solution for (5.24) is

$$
\varphi_{3}(y, \xi, t)=\left.\beta_{1}(\xi+\gamma) D_{+}^{x} D_{y}^{2} p_{0}\right|_{x=-1} e^{-\alpha \xi},
$$

where

$$
\begin{gathered}
\beta_{1}=\frac{1}{2\left(1-e^{-\alpha \triangle \xi}\right)\left(e^{-\alpha \triangle \xi}-e^{\alpha \triangle \xi}\right)}, \quad \gamma=\Delta \xi \frac{e^{-\alpha \triangle \xi}}{1-e^{-\alpha \triangle \xi}} \\
a_{4}^{*}=\frac{1}{2} D_{+}^{\xi} \partial_{t} \varphi_{1}+D_{+}^{\xi} \varphi_{3}, \quad b_{4}^{*}=0 \\
\boldsymbol{u}_{4}^{*}=\boldsymbol{u}_{4}+\frac{1}{2} \partial_{t}^{2} \boldsymbol{u}_{0}+\frac{1}{2} \partial_{t} \nabla_{h} p_{0}, \\
\varphi_{4}=0, \quad a_{5}^{*}=0, \quad b_{5}^{*}=\frac{1}{2} D_{+}^{y} \partial_{t} \varphi_{1}+D_{+}^{y} \varphi_{3} \\
\boldsymbol{u}_{5}^{*}=\boldsymbol{u}_{5}, \\
\left\{\begin{array}{l}
\partial_{t} \boldsymbol{u}_{4}+\nabla_{h} p_{4}=\Delta_{h} \boldsymbol{u}_{4}+\frac{1}{4} \Delta_{h}\left(\partial_{t}^{2} \boldsymbol{u}_{0}+\partial_{t} \nabla_{h} p_{0}\right)-\frac{1}{6} \partial_{t}^{3} \boldsymbol{u}_{0}-\frac{1}{8} \partial_{t}^{2} \nabla_{h} p_{0} \\
\nabla_{h} \cdot \boldsymbol{u}_{4}=0, \\
\boldsymbol{u}_{4}||_{x=-1}=-\left.\frac{1}{2}\left(\partial_{t} \nabla_{h} p_{0}+\frac{1}{2} \partial_{t} \nabla_{\xi} \varphi_{1}\right)\right|_{x=-1, \xi=0} .
\end{array}\right.
\end{gathered}
$$

Now if we let

$$
\left\{\begin{array}{l}
U^{*}=\boldsymbol{u}_{0}^{*}+\sum_{j=1}^{2 N} \varepsilon^{j}\left(\boldsymbol{u}_{j}^{*}+\boldsymbol{a}_{j}^{*}\right), \\
U^{n}=\boldsymbol{u}_{0}+\sum_{j=1}^{2 N} \varepsilon^{j} \boldsymbol{u}_{j}, \\
P^{n-1 / 2}=p_{0}+\sum_{j=1}^{2 N} \varepsilon^{j}\left(p_{j}+\varphi_{j}\right)+\varepsilon^{2 N+1} \varphi_{2 N+1},
\end{array}\right.
$$

then we have

$$
\left\{\begin{array}{l}
\frac{U^{*}-U^{n}}{\Delta t}=\Delta_{h} \frac{U^{*}+U^{n}}{2}+\Delta t^{\alpha} \boldsymbol{f}, \\
U^{*}+U^{n}=\triangle t \nabla_{h} P^{n-1 / 2}, \quad \text { at } \quad x= \pm 1, \\
U^{*}=U^{n+1}+\triangle t \nabla_{h} P^{n+1 / 2}+\triangle t^{\alpha+1} \boldsymbol{g}, \\
\nabla_{h} \cdot U^{n+1}=0, \\
D_{+}^{x} P^{n+1 / 2}=\boldsymbol{n} \cdot U^{n+1}=0, \quad \text { at } \quad x= \pm 1,
\end{array}\right.
$$


where $\alpha=N-1 / 2 ; \boldsymbol{f}$ and $\boldsymbol{g}$ are bounded and smooth if $\left(\boldsymbol{u}_{0}, p_{0}\right)$ is sufficiently smooth. It is easy to see that

$$
\max _{0 \leq t \leq T}\left\|U^{n}(\cdot)\right\|_{W^{1, \infty}} \leq C^{*} .
$$

For the initial approximation, we have

$$
U^{0}(\boldsymbol{x})=\boldsymbol{u}^{0}(\boldsymbol{x})+\triangle t^{2} \boldsymbol{w}^{0}(\boldsymbol{x})
$$

without the extra compatibility condition, and

$$
U^{0}(\boldsymbol{x})=\boldsymbol{u}^{0}(\boldsymbol{x})+\triangle t^{4} \boldsymbol{w}^{0}(\boldsymbol{x})
$$

with the compatibility condition (3.17).

Proof of Theorem 2. Assume a priori that

$$
\max _{0 \leq t^{n} \leq T}\left\|\boldsymbol{u}^{n}\right\|_{W^{1, \infty}} \leq \tilde{C} .
$$

As in the proof of Theorem 1, we let

$$
\boldsymbol{e}^{n}=U^{n}-\boldsymbol{u}^{n}, \quad \boldsymbol{e}^{*}=\hat{U}^{*}-\hat{\boldsymbol{u}}^{*}, \quad q^{n}=P^{n-1 / 2}-p^{n-1 / 2},
$$

where

$$
\begin{aligned}
& 2 \hat{\boldsymbol{u}}^{*}=\boldsymbol{u}^{*}+\boldsymbol{u}^{n}-\triangle t \nabla_{h} p^{n-1 / 2}, \\
& 2 \hat{U}^{*}=U^{*}+U^{n}-\triangle t \nabla_{h} P^{n-1 / 2} .
\end{aligned}
$$

From (5.1) and (5.34), we get

$$
\left\{\begin{array}{l}
\frac{2\left(\boldsymbol{e}^{*}-\boldsymbol{e}^{n}\right)}{\Delta t}+\nabla_{h}\left(q^{n}-\frac{1}{2} \triangle t \Delta_{h} q^{n}\right)=\Delta_{h} \boldsymbol{e}^{*}+\frac{1}{2} \mathcal{N}_{h}\left(\boldsymbol{e}^{n-1}, U^{n-1}\right) \\
\quad+\frac{1}{2} \mathcal{N}_{h}\left(\boldsymbol{u}^{n-1}, \boldsymbol{e}^{n-1}\right)-\frac{3}{2} \mathcal{N}_{h}\left(\boldsymbol{e}^{n}, U^{n}\right)-\frac{3}{2} \mathcal{N}_{h}\left(\boldsymbol{u}^{n}, \boldsymbol{e}^{n}\right)+\triangle t^{\alpha} \boldsymbol{f}^{n}, \\
\boldsymbol{e}^{*}=0, \quad \text { at } \quad x= \pm 1, \\
\frac{\boldsymbol{e}^{n+1}+\boldsymbol{e}^{n}-2 \boldsymbol{e}^{*}}{\triangle t}+\nabla_{h}\left(q^{n+1}-q^{n}\right)=\Delta t^{\alpha} \boldsymbol{g}^{n}, \\
\nabla_{h} \cdot \boldsymbol{e}^{n+1}=0, \\
D_{+}^{x} q^{n+1 / 2}=\boldsymbol{e}^{n+1} \cdot \boldsymbol{n}=0, \quad \text { at } \quad x= \pm 1, \\
\boldsymbol{e}^{0}=\triangle t^{\alpha} \boldsymbol{w}^{0} .
\end{array}\right.
$$

Taking the scalar product of the first equation of (5.40) with $\boldsymbol{e}^{*}$ and integrating by parts, we get

$$
\begin{gathered}
\left\|\boldsymbol{e}^{*}\right\|^{2}-\left\|\boldsymbol{e}^{n}\right\|^{2}+\left\|\boldsymbol{e}^{*}-\boldsymbol{e}^{n}\right\|^{2}+\frac{1}{2} \triangle t\left\|\nabla \boldsymbol{e}^{*}\right\|^{2}+\frac{1}{2} \triangle t\left\|\nabla \cdot \boldsymbol{e}^{*}\right\|^{2} \\
\leq-\triangle t \int_{\Omega} \boldsymbol{e}^{*} \cdot \nabla\left(q^{n}-\frac{1}{2} \triangle t \Delta q^{n}\right) d \boldsymbol{x}+C \triangle t^{2 \alpha+1}\left\|\boldsymbol{f}^{n}\right\|^{2} \\
+C \triangle t\left(\left\|\boldsymbol{e}^{n}\right\|^{2}+\left\|\boldsymbol{e}^{n-1}\right\|^{2}+\left\|\boldsymbol{e}^{*}\right\|^{2}\right)+\frac{1}{2} \triangle t\left\|\nabla \boldsymbol{e}^{*}\right\|^{2}
\end{gathered}
$$


Taking the scalar product of the second equation of (5.42) with $\boldsymbol{e}^{n+1}$, we obtain

$$
\begin{aligned}
& \left\|e^{n+1}\right\|^{2}-\left\|e^{*}\right\|^{2}+\left\|e^{n+1}-e^{*}\right\|^{2}-\frac{1}{2}\left(\left\|e^{n+1}\right\|^{2}-\left\|e^{n}\right\|^{2}\right)-\frac{1}{2}\left\|e^{n+1}-e^{n}\right\|^{2} \\
& \quad \leq C \triangle t^{2 \alpha+1}\left\|g^{n}\right\|^{2}+C \triangle t\left\|e^{n+1}\right\|^{2}
\end{aligned}
$$

Combining these two estimates, we get

$$
\begin{aligned}
\left\|\boldsymbol{e}^{n+1}\right\|^{2}-\left\|\boldsymbol{e}^{n}\right\|^{2}+\left\|\boldsymbol{e}^{n+1}+\boldsymbol{e}^{n}-2 \boldsymbol{e}^{*}\right\|^{2}+\Delta t\left\|\nabla_{h} \boldsymbol{e}^{*}\right\|^{2}+\Delta t\left\|\nabla_{h} \cdot \boldsymbol{e}^{*}\right\|^{2} \\
\leq-2 \triangle t\left(\left(\boldsymbol{e}^{*}, \nabla_{h}\left(q^{n}-\frac{1}{2} \triangle t \Delta_{h} q^{n}\right)\right)\right)+C \triangle t\left(\left\|\boldsymbol{e}^{n}\right\|^{2}+\left\|\boldsymbol{e}^{n-1}\right\|^{2}+\left\|\boldsymbol{e}^{n+1}\right\|^{2}\right) \\
\quad+C \triangle t^{2 \alpha+1}\left(\left\|\boldsymbol{f}^{n}\right\|^{2}+\left\|\boldsymbol{g}^{n}\right\|^{2}\right)
\end{aligned}
$$

To estimate the first term on the right hand side of (5.43), we let

$$
\begin{aligned}
I & \equiv-2 \triangle t\left(\left(e^{*}, \nabla_{h}\left(q^{n}-\frac{1}{2} \Delta t \Delta_{h} q^{n}\right)\right)\right) \\
& =-2 \triangle t\left(\left(e^{*}, \nabla_{h} q^{n}\right)\right)-\Delta t^{2}\left(\left(\nabla_{h} \cdot e^{*}, \Delta_{h} q^{n}\right)\right) \equiv I_{1}+I_{2} .
\end{aligned}
$$

Using the second equation and integrating by parts, we can write the first term as

$$
\begin{aligned}
I_{1}= & -2 \triangle t\left(\left(\boldsymbol{e}^{*}, \nabla_{h} q^{n}\right)\right) \\
= & -\triangle t^{2}\left(\left(\nabla_{h}\left(q^{n+1}-q^{n}\right), \nabla_{h} q^{n}\right)\right)-\triangle t^{\alpha+2}\left(\left(\boldsymbol{g}^{n}, \nabla_{h} q^{n}\right)\right) \\
=- & \frac{1}{2} \triangle t^{2}\left(\left\|\nabla_{h} q^{n+1}\right\|^{2}-\left\|\nabla_{h} q^{n}\right\|^{2}\right) \\
& \quad+\frac{1}{2} \triangle t^{2}\left\|\nabla_{h}\left(q^{n+1}-q^{n}\right)\right\|^{2}-\triangle t^{\alpha+2}\left(\left(\boldsymbol{g}^{n}, \nabla_{h} q^{n}\right)\right) .
\end{aligned}
$$

Since

$$
\begin{aligned}
\frac{1}{2} \triangle t^{2}\left\|\nabla_{h}\left(q^{n+1}-q^{n}\right)\right\|^{2} & =\frac{1}{2}\left\|\boldsymbol{e}^{n+1}+\boldsymbol{e}^{n}-2 \boldsymbol{e}^{*}\right\|^{2} \\
& +\frac{1}{2}\left\|\triangle t^{\alpha+1} \boldsymbol{g}^{n}\right\|^{2}+\triangle t^{\alpha+1}\left(\left(\boldsymbol{g}^{n}, \boldsymbol{e}^{n+1}+\boldsymbol{e}^{n}-2 \boldsymbol{e}^{*}\right)\right),
\end{aligned}
$$

we have

$$
\begin{aligned}
I_{1}= & -\frac{1}{2} \triangle t^{2}\left(\left\|\nabla_{h} q^{n+1}\right\|^{2}-\left\|\nabla_{h} q^{n}\right\|^{2}\right)+\frac{1}{2}\left\|\boldsymbol{e}^{n+1}+\boldsymbol{e}^{n}-2 \boldsymbol{e}^{*}\right\|^{2} \\
& +\frac{1}{2}\left\|\triangle t^{\alpha+1} \boldsymbol{g}^{n}\right\|^{2}+\triangle t^{\alpha+1}\left(\left(\boldsymbol{g}^{n}, \boldsymbol{e}^{n+1}+\boldsymbol{e}^{n}-2 \boldsymbol{e}^{*}\right)\right)-\triangle t^{\alpha+2}\left(\left(\boldsymbol{g}^{n}, \nabla_{h} q^{n}\right)\right) .
\end{aligned}
$$


Next we rewrite the second term as

$$
\begin{aligned}
I_{2}= & -\triangle t^{2}\left(\left(\nabla_{h} \cdot \boldsymbol{e}^{*}, \Delta_{h} q^{n}\right)\right) \\
= & -\frac{1}{2} \triangle t^{3}\left(\left(\Delta_{h}\left(q^{n+1}-q^{n}\right), \Delta_{h} q^{n}\right)\right)-\frac{1}{2} \triangle t^{\alpha+3}\left(\left(\nabla_{h} \cdot \boldsymbol{g}^{n}, \Delta_{h} q^{n}\right)\right) \\
= & -\frac{1}{4} \triangle t^{3}\left(\left\|\Delta_{h} q^{n+1}\right\|^{2}-\left\|\Delta_{h} q^{n}\right\|^{2}\right)+\frac{1}{4} \triangle t^{3}\left\|\Delta_{h}\left(q^{n+1}-q^{n}\right)\right\|^{2} \\
& \quad-\frac{1}{2} \triangle t^{\alpha+3}\left(\left(\nabla_{h} \cdot \boldsymbol{g}^{n}, \Delta_{h} q^{n}\right)\right) \\
= & -\frac{1}{4} \triangle t^{3}\left(\left\|\Delta_{h} q^{n+1}\right\|^{2}-\left\|\Delta_{h} q^{n}\right\|^{2}\right)+\triangle t\left\|\nabla_{h} \cdot \boldsymbol{e}^{*}\right\|^{2}+\frac{1}{4} \triangle t^{2 \alpha+3}\left\|\nabla_{h} \cdot \boldsymbol{g}^{n}\right\|^{2} \\
& -\triangle t^{\alpha+2}\left(\left(\nabla_{h} \cdot \boldsymbol{g}^{n}, \nabla_{h} \cdot \boldsymbol{e}^{*}\right)\right)-\frac{1}{2} \triangle t^{\alpha+3}\left(\left(\nabla_{h} \cdot \boldsymbol{g}^{n}, \Delta_{h} q^{n}\right)\right) .
\end{aligned}
$$

Combining these two terms, we arrive at

$$
\begin{aligned}
I= & -\frac{1}{2} \triangle t^{2}\left(\left\|\nabla_{h} q^{n+1}\right\|^{2}-\left\|\nabla_{h} q^{n}\right\|^{2}\right)-\frac{1}{4} \triangle t^{3}\left(\left\|\Delta_{h} q^{n+1}\right\|^{2}-\left\|\Delta_{h} q^{n}\right\|^{2}\right) \\
& +\frac{1}{2}\left\|\boldsymbol{e}^{n+1}+\boldsymbol{e}^{n}-2 \boldsymbol{e}^{*}\right\|^{2}+\triangle t\left\|\nabla_{h} \cdot \boldsymbol{e}^{*}\right\|^{2}+\triangle t^{\alpha+1}\left(\left(\boldsymbol{g}^{n}, \boldsymbol{e}^{n+1}+\boldsymbol{e}^{n}-2 \boldsymbol{e}^{*}\right)\right) \\
& -\triangle t^{\alpha+2}\left(\left(\boldsymbol{g}^{n}, \nabla_{h} q^{n}\right)\right)-\triangle t^{\alpha+2}\left(\left(\nabla_{h} \cdot \boldsymbol{g}^{n}, \nabla_{h} \cdot \boldsymbol{e}^{*}\right)\right) \\
& -\frac{1}{2} \triangle t^{\alpha+3}\left(\left(\nabla_{h} \cdot \boldsymbol{g}^{n}, \Delta_{h} q^{n}\right)\right)+\frac{1}{4} \triangle t^{2 \alpha+3}\left\|\nabla_{h} \cdot \boldsymbol{g}^{n}\right\|^{2}+\frac{1}{2}\left\|\triangle t^{\alpha+1} \boldsymbol{g}^{n}\right\|^{2}
\end{aligned}
$$

This gives

$$
\begin{aligned}
I \leq & -\frac{1}{2} \triangle t^{2}\left(\left\|\nabla_{h} q^{n+1}\right\|^{2}-\left\|\nabla_{h} q^{n}\right\|^{2}\right)-\frac{1}{4} \triangle t^{3}\left(\left\|\Delta_{h} q^{n+1}\right\|^{2}-\left\|\Delta_{h} q^{n}\right\|^{2}\right) \\
& +\frac{1}{2}\left\|\boldsymbol{e}^{n+1}+\boldsymbol{e}^{n}-2 \boldsymbol{e}^{*}\right\|^{2}+\triangle t\left\|\nabla_{h} \cdot \boldsymbol{e}^{*}\right\|^{2}+\triangle t\left\|\boldsymbol{e}^{n+1}+\boldsymbol{e}^{n}-2 \boldsymbol{e}^{*}\right\|^{2} \\
& +2 \triangle t^{3}\left\|\nabla_{h} q^{n}\right\|^{2}+2 \triangle t^{4}\left\|\Delta_{h} q^{n}\right\|^{2}+2 \triangle t^{2 \alpha+1}\left(\left\|\boldsymbol{g}^{n}\right\|^{2}+\triangle t\left\|\boldsymbol{g}^{n}\right\|_{H^{1}}^{2}\right) .
\end{aligned}
$$

Going back to (5.45), we obtain

$$
\begin{aligned}
\| e^{n+1} & \left\|^{2}-\right\| e^{n}\left\|^{2}+\frac{1}{2}\right\| e^{n+1}+e^{n}-2 e^{*}\left\|^{2}+\triangle t\right\| \nabla_{h} e^{*} \|^{2} \\
& +\frac{1}{2} \triangle t^{2}\left(\left\|\nabla_{h} q^{n+1}\right\|^{2}-\left\|\nabla_{h} q^{n}\right\|^{2}\right)+\frac{1}{4} \triangle t^{3}\left(\left\|\Delta_{h} q^{n+1}\right\|^{2}-\left\|\Delta_{h} q^{n}\right\|^{2}\right) \\
\leq & \triangle t^{3}\left\|\nabla_{h} q^{n}\right\|^{2}+\triangle t^{4}\left\|\Delta_{h} q^{n}\right\|^{2}+C \triangle t\left(\left\|e^{n}\right\|^{2}+\left\|e^{n-1}\right\|^{2}+\left\|e^{n+1}\right\|^{2}\right) \\
& +C \triangle t^{2 \alpha+1}\left(\left\|\boldsymbol{f}^{n}\right\|^{2}+\triangle t\left\|\boldsymbol{g}^{n}\right\|_{H^{1}}^{2}\right) .
\end{aligned}
$$

Gronwall's lemma gives

$$
\left\|\boldsymbol{e}^{n}\right\|+\left\|\boldsymbol{e}^{*}\right\|+\triangle t\left\|\nabla_{h} q^{n}\right\|+\triangle t^{3 / 2}\left\|\Delta_{h} q^{n}\right\|+\triangle t^{1 / 2}\left\|\nabla_{h} \boldsymbol{e}^{*}\right\| \leq C_{1} \triangle t^{\alpha} .
$$

Now by the inverse inequality (4.4) we have

$$
\left\|\boldsymbol{e}^{n}\right\|_{L^{\infty}}+h\left\|\boldsymbol{e}^{n}\right\|_{W^{1, \infty}}+\triangle t\left\|\nabla_{h} q^{n}\right\|_{L^{\infty}} \leq C_{1} \frac{\triangle t^{\alpha}}{h} .
$$


If $N=5$ and $\Delta t^{\alpha}<<h^{2}$, and we choose $\triangle t$ small enough, we will always have

$$
\left\|e^{n+1}\right\|_{L^{\infty}} \leq 1
$$

Therefore in (5.39) we can choose

$$
\tilde{C}=1+\max _{n \leq\left[\frac{T}{\Delta t}\right]}\left\|U^{n}(\cdot)\right\|_{W^{1, \infty}},
$$

which depends only on the exact solution $(\boldsymbol{u}, p)$. This proves that

$$
\left\|\boldsymbol{u}_{0}-\boldsymbol{u}_{h}\right\|_{L^{\infty}}+\left\|p_{0}-p_{h}\right\|_{L^{2}}+\triangle t^{1 / 2}\left\|p_{0}-p_{h}\right\|_{L^{\infty}}+\left\|p_{0}-p_{h}-p_{c}\right\|_{L^{\infty}} \leq C \triangle t^{2} .
$$

From Lemma 4.2, we have

$\left\|\boldsymbol{u}-\boldsymbol{u}_{h}\right\|_{L^{\infty}}+\left\|p-p_{h}\right\|_{L^{2}}+\triangle t^{1 / 2}\left\|p-p_{h}\right\|_{L^{\infty}}+\left\|p-p_{h}-p_{c}\right\|_{L^{\infty}} \leq C\left(\triangle t^{2}+h^{2}\right)$.

This completes the proof of Theorem 2 .

\section{Conclusions}

The main conclusion from the analysis presented in this paper is that the numerical error in the projection method has the same structure as in the semi-discrete case (analyzed in 4]) after it is spatially discretized on a staggered grid. This paper leaves open the very interesting question of what happens on a regular nonstaggered grid. Some progress in this direction has been made in [16].

\section{ACKNOWLEDGMENTS}

The research of the first author was supported in part by ONR grant N0001496-1-1013 and NSF grant DMS-9623137. The research of the second author was supported in part by ONR grant N00014-96-1-1013 and NSF grants DMS-9505275 and DMS-9805621.

\section{REFERENCES}

1. J.B. Bell, P. Colella, and H.M. Glaz, A second-order projection method for the incompressible Navier Stokes equations, J. Comput. Phys. 85 (1989), 257-283. MR 90i:76002

2. A.J. Chorin, Numerical solution of the Navier-Stokes equations, Math. Comp. 22 (1968), 745-762. MR 39:3723

3. Weinan E and J.-G. Liu, Projection method I: convergence and numerical boundary layers, SIAM J. Numer. Anal. 32 (1995), 1017-1057; Projection method II: Godunov-Ryabenki analysis, SIAM J. Numer. Anal. 33 (1996), 1597-1621. MR 96e:65061 MR 97i:76078

4. Weinan E and J.-G. Liu, Projection method for viscous incompressible flows, in "Numerical Methods in Applied Sciences", edited by W. Cai and C.-W. Shu, Science Press New York, Ltd, New York, (1996), 78-96

5. Weinan E and J.-G. Liu, Gauge method for viscous incompressible flows, preprint, (1996)

6. M. Fortin, R. Peyret, and R. Temam, Résolution numérique des équations de Navier-Stokes pour un fluide incompressible, J. Mecanique, 10 (1971) 357-390. MR 54:9343

7. F.H. Harlow and J.E. Welch, Numerical calculation of time-dependent viscous incompressible flow of fluid with free surface, Phys. Fluids, 8 (1965) 2182-2189.

8. J. Heywood and R. Rannacher, Finite element approximation of the nonstationary NavierStokes problem, part III. Smoothing property and higher order error estimates for spatial discretization, SIAM J. Numer. Anal., 25, (1988) 490-512. MR 89k:65114

9. J. Kim and P. Moin, Application of a fractional-step method to incompressible Navier-Stokes equations, J. Comp. Phys. 59 (1985), 308-323. MR 87a:76046

10. J. Shen, On error estimates of projection methods for Navier-Stokes equations: first order schemes, SIAM J. Numer. Anal. 29 (1992), 57-77. MR 92m:35213 
11. J. Shen, On error estimates of some higher order projection and penalty-projection methods for Navier-Stokes equations, Numer. Math. 62 (1992), 49-73. MR 93a:35122]

12. R. Temam, Sur l'approximation de la solution des equations de Navier-Stokes par la méthode des fractionnarires II, Arch. Rational Mech. Anal. 33 (1969), 377-385. MR 39:5968

13. J. van Kan, A second-order accurate pressure-correction scheme for viscous incompressible flow, SIAM J. Sci. Stat. Comp. 7 (1986), 870-891. MR 87h:76008

14. C. Wang and J.-G. Liu, Convergence of gauge method for incompressible flow, Math. Comp., 69 (2000), 1385-1407. MR 20001a:65103

15. B.R. Wetton, Ph.D Dissertation, New York University, 1991.

16. B.R. Wetton, Error analysis for Chorin's original fully discrete projection method and regularizations in space and time, SIAM J. Numer. Anal. 34 (1997), 1683-1697. MR 99c:65161

Courant Institute of Mathematical Sciences, New York, New York 10012

E-mail address: weinan@cims.nyu.edu

Institute for Physical Science and Technology and Department of Mathematics, University of Maryland, College Park, Maryland 20742

E-mail address: jliu@math.umd.edu 